\title{
A Method of Enhanced Corner Points Extraction Based on Circular Neighborhood
}

\author{
Zetao Jiang, Xing Yu, and Le Zhou
}

\begin{abstract}
In this paper, it presents an improved Harris feature extraction algorithm, which is based on Harris algorithm to extract candidate feature points and screen angular points that satisfy certain constraints on the edge of circular neighborhood for the determination of final feature points. And then it makes use of Gaussian-weighted to establish a proximity matrix between internal feature points in two images and uses the singular value decomposition to get their eigenvectors. At last it makes use of eigenvectors to create a relation matrix to reflect the matching degree between feature points for matching. And it shows that extracted feature points in the proposed method in the experiments is robust strongly for rotation, illumination, noise, etc., with good matching effect, low rate of false matches.
\end{abstract}

Index Terms-Harris, the edge of circular neighborhood, singular value decomposition.

\section{INTRODUCTION}

Image matching algorithm, widely applied in the field of image recognition and image reconstruction, is one of the key steps to achieve image fusion and image mosaic. And image matching has an important part that is how to extract reliable feature points and improve the matching accuracy. Generally, image matching composes of two categories: matching based on gray scale [1], [2] and that on feature points [3]-[5]. Based on gray scale, the matching method directly calculates the difference value of pixel gray-scale between two images. It is relatively easy, yet particularly time-consuming. At the same time, in the case of a large rotation, light, partial occlusion and random noise, the results obtained by an image are not good. While based on feature points, including Harris operator [6], [7], SIFT feature point, SURF feature point and so on, the matching method whose performance is depended largely on the quality of extraction features can better solve such problems. In the current feature point matching algorithms, most detection methods of feature points are to consider the $n \times n$ square neighborhood. When more adjacent feature points are extracted, it is often prone to mismatching. If these mismatching occur during the image mosaic, it will bring many ghost shadows to mosaic images. So in order to avoid such conditions, the number of image feature points must be not too large or too small. In this paper, it will select the Harris feature extraction algorithm to extract angular points as pending feature points, then Shapiro and Brady algorithm

Manuscript received January 5, 2014; revised March 25, 2014

Zetao Jiang is with Guilin University of Electronic Technology, Guilin, China (e-mail: zetaojiang@126.com).

Xing Yu and Le Zhou are with Nanchang Hangkong University, School of information and engineering, Nan Chang, China (e-mail: 258371880@qq.com, 450702826@qq.com).
[8], [9] that is one of Spectral Graph theory will be used to match the images. It makes use of Gaussian-weighted to establish a proximity matrix between internal feature points in two images and uses the singular value decomposition[10]-[14] to get their eigenvectors. Then it makes use of eigenvectors to create a relation matrix reflecting the matching degree between feature points for matching. Finally, false matching point-pair will be eliminated through RANSAC algorithm.

\section{FUNDAMENTALS OF ALGORITHMS}

\section{A. Harris Algorithm}

Developed on the basis of Moravec Algorithm [15], Harris angular point detection algorithm is a detection operator based on point features of signals. Its principle is to select a small window centered by target pixels and move the window along any direction to compute gray scale change in any direction within the window. Its analysis formula is shown as follows:

$$
\begin{aligned}
& E_{x y}=\sum \omega_{x, y}\left(I_{x|v, y| v}-I_{x, y}\right)^{2} \\
& =\sum \omega_{x, y}\left\{v \frac{\partial I}{\partial X}+v \frac{\partial I}{\partial Y}+o\left(\sqrt{v^{2}+v^{2}}\right)\right\}^{2}
\end{aligned}
$$

In order to improve noise immunity, Gaussian smoothing is applied to the image window and one Gaussian window is chosen:

$$
W_{v, y}=\ell\left[-\frac{\frac{1}{2}\left(v^{2}+v^{2}\right)}{\sigma^{2}}\right]
$$

In the Harris algorithm, an autocorrelation matrix is used to screen the angular points and partial derivatives are used to create a matrix,

$$
H=\left[\begin{array}{ll}
I_{x x} & I_{x y} \\
I_{x y} & I_{y y}
\end{array}\right]
$$
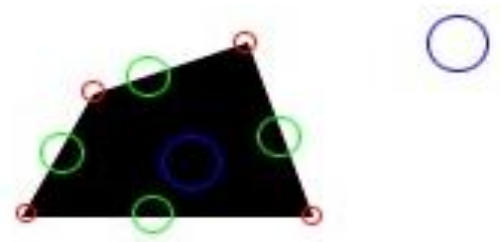

Fig. 1. Three cases of image points.

As shown in Fig. 1, points can generally be divided into 
three kinds: edge points (in green), flat area points (in blue) and angular points (in red).

First, the three cases are judged by eigenvalues:

1) Two small directional curvatures, indicating local autocorrelation function is flat;

2) One big directional curvature and one relatively small, showing here has an edge;

3) Two big directional curvatures, meaning that local autocorrelation function has a spike which is an angular point.

The ratio of matrix eigenvalue $H$ and $f$ are obtained to remove unstable edge points and their ratio formula is

$$
\begin{gathered}
\operatorname{Tr}(H)=I_{x x}+I_{y y}=\alpha+\beta \\
\operatorname{Det}(H)=I_{x x} I_{y y}-\left(I_{x y}\right)^{2}=\alpha \beta
\end{gathered}
$$

Among which, $\alpha$ is a big eigenvalue of $H$ and $\beta$ is a small eigenvalue.

$$
\frac{\operatorname{Tr}(H)^{2}}{\operatorname{Det}(H)}=\frac{(\alpha+\beta)^{2}}{\alpha \beta}=\frac{(r+1)^{2}}{r}
$$

The formula of Harris Corner Response Function is:

$$
C R F(x, y)=\operatorname{det}(M)-k(\operatorname{trace}(M))
$$

\section{B. Shapiro and Brady Algorithm Principles}

Matrix H structured by points in one image is symmetric, so SVD decomposition can be used to obtain $D$, and elements on the $D$ Diagonal are eigenvalues of Matrix $H$, which are arranged in descending. In the formula $H=V D U$, after its decomposition, $V$ whose structure is an inherent feature of the matrix, represents eigenvectors of each array. Therefore, it is a modal matrix and is orthogonal. While in the $V=\left[E_{1} E_{2} \cdots E_{n}\right], E_{1}$ means the $i$ eigenvector in Matrix $H$, each line of which can be considered as Vector $F_{i}$, indicating its features and it is the modal coordinates of the $i$ feature point. So the feature point set of an image in $m$ modal coordinates can be expressed as: $V=\left(F_{1} F_{2} \cdots F_{\mathrm{m}}\right)^{T}$. And then $S V D$ decomposition is separately applied to two images to find eigenvectors of these two images as $F_{i 1}$ and $F_{i 2}$. Finally, these two groups of eigenvectors are used to form a relationship matrix $Z$, which shows the matching degree between two points. In addition, it is quite remarkable that the different quantity of feature points included in the image feature point set leads to various quantity of models. Therefore, a less important model $|m-n|$ needs to be removed from an image with more feature points. Meanwhile, these eigenvalues corresponded to modes are relatively small, and symbols of each eigenvector are not unique. But it will not affect the orthogonality when its symbol is changed. Because two axes must have the same direction, it is necessary to modify symbols. First, determining $V_{x}$ and then modify eigenvectors in $V_{y}$ based on that in $V_{x}$ to makes two vectors consistent. Only in this way the formula $Z_{i j}=\left\|F_{i 1}-F_{j 2}\right\|^{2}$ makes sense.

\section{The Algorithm PROCESS}

\section{A. Improved Harris Detection Algorithm}

Firstly, the process of Harris method can be divided into the following steps:

To calculate gradients of image pixels in the horizontal and vertical directions as well as their product for obtaining $H$

$$
H=\left[\begin{array}{ll}
I_{x x} & I_{x y} \\
I_{x y} & I_{y y}
\end{array}\right]
$$

1) Gaussian filter applied to the image for a new Matrix $M$

2) To calculate corresponding interest values of each pixel in the original image

3) To select local extreme points.

In the Harris algorithm, the determination of an angular point is based on the condition where the value of a pixel is bigger than a given threshold value and it is the maximum value in a $3 \times 3$ neighborhood. In this case, it is an angular point. When a $3 \times 3$ neighborhood is used to do image processing, it is likely to present relatively intensive feature points on a local scale and easy to show mismatching. Moreover, further points in the neighborhood will also have an influence on it. When a $3 \times 3$ neighborhood is used to make a judgment, edge pixels in a circular with a radius of 3 can also be used. Originally, it was compared with eight points next to it and now with sixteen points around it to reduce the aggregation of angular points on the local scale. Hence, a circular distinguishing method in this paper is used to enhance the stability of feature points:

$$
\left\{\begin{array}{l}
I(x)-I(q+1)<\varepsilon d \\
I(x)-I(q-1)<\varepsilon d
\end{array}\right.
$$

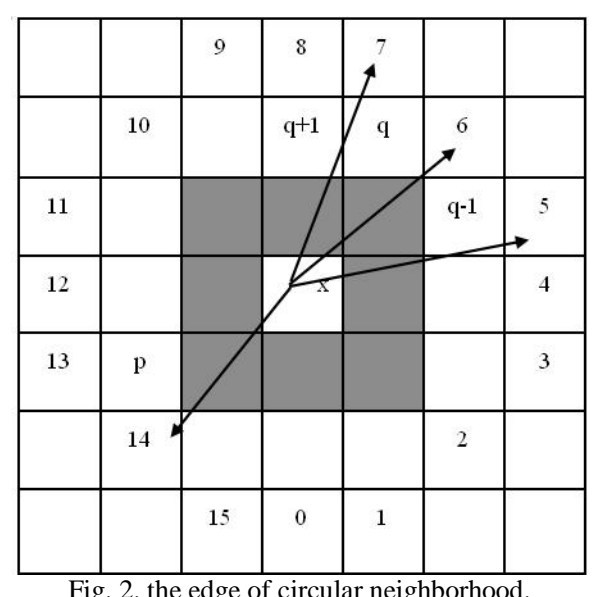

In addition to the use of feature points in a $3 \times 3$ neighborhood to determine a stable angular point, it also uses the above formula for surrounding points removal. That is, it is not only considered as an angular point determined by Harris Corner Response Function, but also an angular point in a circular neighborhood. Only in this case can it be a stable feature point. Since the two parts are carried out simultaneously, it does not increase too much calculation time. As shown in Fig. 2 below, $q+1$ and $q-1$ are symmetrical about point $q$, meaning it must be guaranteed 
there are 6 pixels satisfying the above discriminant for any pixel in this circular neighborhood. If not, then the central point is not an angular point but an edge point that need removing. The threshold value varies with conditions, showing the minimum contrast of detection feature points and the maximum limit of ignoring noise. According to images with different noises, it will get different values to control the number of extracted feature points.

\section{B. Feature Point Matching Based on Singular Value}

Assuming that the extraction of feature points in the first image $S_{1}$ in a total of $m, d_{1}$ is the feature points No. $i$, with its main direction $\theta_{1}$ and coordinates $\left(x_{1}, y_{1}\right)$, the extraction of feature points in the second image $S_{2}$ in a total of $n, d_{2}$ is the feature points No. $j$, with its main direction $\theta_{2}$ and coordinates $\left(x_{2}, y_{2}\right)$, the central rectangular window of the two feature points are $W_{1}$ and $W_{2}$ respectively and their size are $(2 w+1) \times(2 w+1)$.

To establish a proximity matrix $H$ for the two images using the method of Gaussian-weighted, among which:

$$
H_{i j}=\ell^{\left(-\frac{d_{i j}^{2}}{2 \sigma_{x}^{2}}\right)} \quad i=1 \cdots n, j=1 \cdots n
$$

where $d_{i j}=\left\|x_{i}-x_{j}\right\|$, and it represents Euclidean distance of own internal feature points in the image, $\sigma$ represents the effect of controlling the distance between the feature points, $x$ represents that the similarity measurements are in the same feature point set. After getting their proximity matrixes, the two sensitivity matrixes are decomposed by singular value decomposition (SVD) method to get $H_{1}=V_{1} D_{1} U_{1}$, and then use the identity matrix whose $H_{2}=V_{2} D_{2} U_{2}$ is $m \times n$ instead of matrix $D$, in this way, the matrix $P=V E U$ obtained. Since matrix $V$ is orthogonal, each line of $V$ can be regarded as a eigenvector, using $V_{1}$ to establish a relation matrix $Z$ of $m \times$ $n$,

$$
Z_{i j}=\left\|F_{i 1}-F_{j 2}\right\|^{2}
$$

The $F_{i 1}$ and $F_{j 2}$ in this formula represents the $i$ row vector in $V_{1}$ and $j$ column vector in $V_{2}$ respectively, $Z_{i j}$ stands for the matching degree of two feature points, the smaller the $Z_{i j}$ value is, the more matching the feature points are. If the value reaches 0 , the two feature points are totally matching.

\section{OUR AlgorithM STEPS}

1) To extract rough feature angular point by using the autocorrelation matrix in 2.1 ;

2) Then, to gain stable angular point by using neighborhood edge restriction;

3) To match the feature points based on the singular value decomposition in 2.3;

4) To eliminate false matching and get the final fine matching by using RANSAC algorithm.

\section{ANALYSIS OF EXPERIMENTS AND RESULTS}

This paper uses Matlab to implement the algorithm, and compares the proposed Harris-SVD algorithm with improved Harris-SVD algorithm on the image matching performance and analyzes it.

The images in Fig. 3(a) are just matched by Harris-SVD, while the images in Fig. 3(b) matched by Harris-SVD, combining using circular neighborhood to limit and eliminate some unstable angular points, and Fig. 3(c) uses RANSAC algorithm to implement the final fine matching.

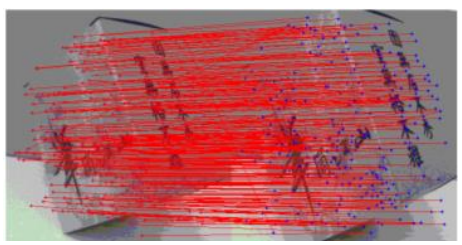

(a)

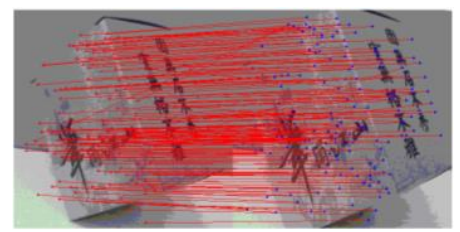

(b)

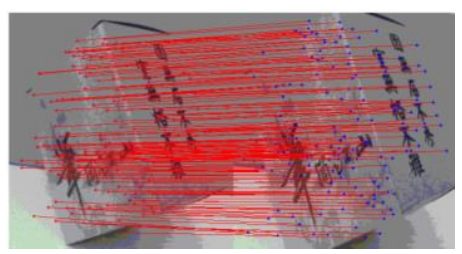

(c)

Fig. 3. Images of our experiment.

TABLE I: THE COMPARES OF OUR ALGORITHM AND HARRIS-SVD
\begin{tabular}{|c|c|c|c|c|}
\hline Methods & $\begin{array}{c}\text { Feature } \\
\text { points }\end{array}$ & $\begin{array}{c}\text { Initial } \\
\text { matched } \\
\text { pairs }\end{array}$ & $\begin{array}{c}\text { Correct } \\
\text { points } \\
\text { pairs }\end{array}$ & $\begin{array}{c}\text { The rate } \\
\text { of wrong } \\
\text { matched } \\
\text { pairs }\end{array}$ \\
\hline Harris-SVD & 957 & 335 & 258 & $23 \%$ \\
\hline $\begin{array}{c}\text { Improved } \\
\text { Harris-SVD }\end{array}$ & 678 & 235 & 186 & $21 \%$ \\
\hline
\end{tabular}

As can be seen in the Table I, there are more angular points after simply Harris screening in the collected image. Although there are more matching points, reaching to 335 pairs, there are more false matching points and false match rate is higher. While adding the limit of circular neighborhood, the resulting feature points are more balanced. Although the feature point pairs are fewer, the images eliminate some unstable points and leave more stable feature points.

\section{CONCLUSION}

This paper puts forward an improved Harris-SVD feature matching algorithm, introducing the edge of circular neighborhood into Harris detection algorithm to handle the phenomenon of prone to false matches when the gotten points aggregate in the multi-area. From the experiments, we got that more feature points may result in more incorrect matching, especially the distance of the location of some points is too small. Therefore the two judgments are obtained to limit the number of feature points. Since the two judgments are implemented at the same time, we can get more stable angular points and reduce the probability of false matches without increasing the computation. Besides, 
our algorithm also has rotational invariance, and a stronger robustness to partial occlusion and illumination changes.

\section{ACKNOWLEDGE}

This paper is sponsored by Nature Science Foundation of China (61272216) and innovation Foundation of aerospace in China (CASC201102).

\section{REFERENCE}

[1] J. Gao, J. Y. Sun, and J. Liu, "Image matching method based on normalized grayscale variance Hausdorff distance," Journal of computer Applications, vol. 31, no. 3, 2011.

[2] J. J. Li, L. Zhang, X. G. Li, X. Chen, and Y. S. Huang, "Parallel grey-level image matching algorithm with CUDA," Journal of University of Electronic Science and Technology of China, vol. 41, no. 1,2012 .

[3] T. Q. Han, Y. D. Zhao, S. L. Liu, and Y. Bai, "Spatially constrained SURF feature point matching for UAV images," Journal of Image and Graphics, vol. 18, no. 6, 2013.

[4] G. J. Chen, Z. Q. Ma, Y. Shan, and X. Y. Zhang, "Fast image registration method based on local salient feature," Application Research of Computers, vol. 29, no. 11, 2012.

[5] H. C. Yang, G. B. Yao, and Y. B. Wang, "Dense matching for wide base-line stereo images based on SIFT," Acta Geodaetica et Cartographica Sinica, vol. 40, no. 5, 2011.

[6] X. D. Lv and Z. J. Wang, "Perceptual image hashing based on shape contexts and local feature points," IEEE Transactions Information Forensics and Security, vol. 7, 2012.

[7] A. Das and D. Ghoshal, "Human eye detection of color images based on morphological segmentation using modified Harris corner detector," in Proc. 2012 International Conference on Emerging Trends in Electrical Engineering and Energy Management (ICETEEEM), IEEE, 2012, pp. 143-147.

[8] J. Tang, T. Gao, and D. Liang, "Point pattern matching: spectral descriptor based approach," Journal of Computer-Aided Design and Computer Graphics, vol. 25, no. 9, 2013.

[9] Z. P. Yu and X. M. Li, "Based on spectral graph theory of feature matching principle research," Journal of Taiyuan Normal University, vol. 11, no. 2, 2012.

[10] S. Arora, R. Ge, and A. Moitra, "Learning topic models--going beyond SVD," in Proc. 2012 IEEE 53rd Annual Symposium on Foundations of Computer Science (FOCS), IEEE, 2012, pp. 1-10.

[11] R. Rubinstein, T. Faktor, and M. Elad, "K-svd dictionary-learning for the analysis sparse model," in Proc. 2012 IEEE International Conference on Acoustics, Speech and Signal Processing (ICASSP), IEEE, 2012, pp. 5405-5408.

[12] J. A. Bullinaria and J. P. Levy, "Extracting semantic representations from word co-occurrence statistics: stop-lists, stemming, and SVD," Behavior Research Methods, vol. 44, no. 3, pp. 890-907, 2012.
[13] L. Hong, H. Lu, and X. Xue, "A Segmentation and graph-based video sequence matching method for video copy detection," IEEE Transactions on Knowledge and Data Engineering, vol. 25, 2013.

[14] F. Zhao and Q. M. Huang, "An image matching algorithm based on singular value decomposition," Journal of Computer Research and Development, 2010, vol. 47, no. 1, pp. 23-32.

[15] Y. Lu, "Research on Moravec and Harris corner algorithm," Computer Technology and Development, vol. 21, no. 6, 2011.

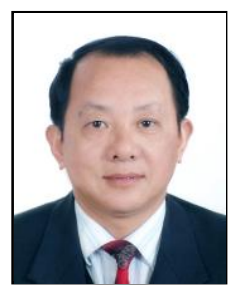

Zetao Jiang was born in 1961 in Jiangxi Province. $\mathrm{He}$ is an adjunct professor of Guilin Uniersity of Electronic Technology, also a PhD supervisor and an academic leader in Guangxi province. He received the B.S. degree in Beijing Normal University, Beijing, China, in 1986. He received the M.S. degree in Tongji University, Shanghai, China, in 1995 and received the doctor of philosophy in Northwestern Polytechnical University, Xi'an, China, in 2006. His main activities were research in the field of image processing and computer vision.

He presided key technology research project "Control system based on multi-level security agent", which was awarded the second class prize in the 2009 annual science and technology progress of Jiangxi province. He presided the research of network and information security defense technology based on digital watermarking, which was awarded the first class prize in science and technology achievements of Jiangxi province. He presided and finished Nature Science Foundation of China (60673055), two subject Foundations of province, an open foundation of measurement and control center of province and more than 10 other subjects. Currently, he is presiding Nature Science Foundation of China (60673055) and Nature Science Foundation of Province, which is in the field of 3D reconstruction.

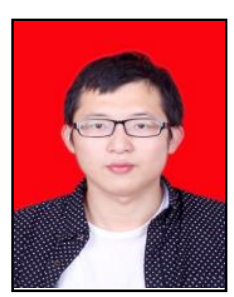

Xing Yu was born in 1989 in Jiangxi Province, and had received the B.S degree in Guilin University of Electronic Technology. Currently, he is also pursuing the M.S degree in Nanchang Hangkong University, and his research area includes image processing and computer vision

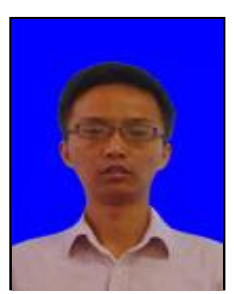

Le Zhou was born in 1988 in Jiangsu Province, and received the B.S degree in Yancheng Teachers' College, Yancheng, China. Currently, he is pursuing the M.S degree in Nanchang Hangkong University, and the main research area includes image processing and computer vision. 